\title{
Inelastic Neutron Scattering Investigation of Magnetic Excitations in the Quenched and Decomposed Samples of the $\mathrm{Mn}_{0.75} \mathrm{Cu}_{0.25}$ Alloy
}

\author{
J. JANKOWSKA-KISIELIŃSKA* AND K. ŚWIDERSKA \\ National Centre for Nuclear Research (NCBJ), A. Sołtana 7, 05-400 Otwock-Świerk, Poland
}

Dedicated to the Memory of Kazimierz Mikke

\begin{abstract}
The influence of spinodal decomposition on the spin wave characteristics in the $\mathrm{Mn}_{0.75} \mathrm{Cu}_{0.25}$ alloy was studied with inelastic neutron scattering. The measurements were performed on the quenched and annealed samples at a few temperatures. Parameters of spin-wave dispersion-relation for small wave-vector were derived. The values of spin wave velocity and energy gap obtained at reduced temperature $T / T_{\mathrm{N}} \approx 0.65$ are after decomposition twice higher than for the quenched sample. For the decomposed sample the values of both parameters are close to the results published for the $\mathrm{Mn}_{0.9} \mathrm{Cu}_{0.1}$ alloy. This fact confirms that after decomposition spin waves are present in the sample regions of $\mathrm{Cu}$ concentration close to 0.1 and that the neighborhood of regions with high $\mathrm{Cu}$ content seems not to influence them. The spin wave velocity for the quenched sample is lower than observed in any other manganese alloy.
\end{abstract}

DOI: 10.12693 /APhysPolA.124.990

PACS: 61.05.fm, 78.70.Nx, 75.30.Ds, 81.40.-z

\section{Introduction}

Annealing at $400-600 \mathrm{~K}$ is well known to cause phase decomposition of the $\mathrm{Mn}_{1-x} \mathrm{Cu}_{x}$ alloys for $x<0.5$ $[1,2]$. Two phases with different $\mathrm{Cu}$ content are formed: one with $x<0.1$, and the other with $x$ close to 0.6. Our understanding of the phase separation process in manganese-copper alloys has been now strongly enhanced by detailed study on $\mathrm{Mn}_{0.67} \mathrm{Cu}_{0.33}$ [3] that showed that the kinetics of phase separation is well described by spinodal decomposition theory at early-time stage and by dynamical scaling at late-time stage of decomposition.

The influence of decomposition on magnetic structure was investigated earlier only above room temperature $[1,2,4-6]$. Our previous investigations on the quenched and decomposed samples of the $\mathrm{Mn}_{0.75} \mathrm{Cu}_{0.25}$ alloy [7] revealed the diffuse neutron scattering at the $(1,1 / 2,0)$ reciprocal lattice point (RLP) with similar intensity below $50 \mathrm{~K}$ in both samples. These observations indicate that, in addition to the well known F1 antiferromagnetic long range order, in both samples there is a short range order (SRO) of type different from F1. In the quenched sample this SRO has magnetic character. In the decomposed sample the $\mathrm{F} 1$ order is present in the regions of low $\mathrm{Cu}$ content, and in the regions of high $\mathrm{Cu}$ content the SRO of both atomic and magnetic character is present. The subject of the present inelastic neutron scattering study was spin waves occurring in the F1 phase in both samples.

* corresponding author; e-mail:

j.jankowska-kisielinska@ncbj.gov.pl
Spin waves in $\mathrm{Mn}_{1-x} \mathrm{Cu}_{x}$ alloys were investigated earlier in quenched samples with $x=0.1[8,9], x=0.15$ [10] and $x=0.18[11,12]$. The spin wave (SW) dispersion relation for an antiferromagnetic with anisotropic interaction is known to have for a small wave-vector the simple form (e.g. [8]):

$$
E^{2}(q)=E_{\mathrm{g}}^{2}+(v q)^{2},
$$

where $E_{\mathrm{g}}=E(q=0)$ is the energy gap of the SW spectrum, and $v$ is the SW velocity. The published data indicate that values of the both dispersion-relation parameters fall with Mn concentration decreasing.

Since 1973 Gillan's paper [13] spin waves in manganese alloys are understood in terms of the electron band model. In simplification of this model the SW velocity is proportional to the "effective" Fermi velocity of electrons from bands forming magnetization. Alloying can influence SW velocity in two ways: by changing the Fermi velocity and introducing scattering of SW on impurities.

The energy gap in SW spectrum is interpreted in terms of the magneto-elastic interaction [14] which induces tetragonal distortion of the lattice. In this model SW energy gap is proportional to the gap in quasiparticle energy band produced by exchange interaction in antiferromagnetic phase. The value of the band gap is strongly dependent on the sub-lattice magnetization and on shape and dimensions of the Fermi surface. In consequence alloying influences on the value of energy gap by changing Fermi surface.

\section{Experimental details}

Three $1 \mathrm{~cm}^{3}$ single-crystal cubic samples of $\mathrm{Mn}_{0.75} \mathrm{Cu}_{0.25}$ alloy were homogenized by annealing at $\approx 1100 \mathrm{~K}$ and quenching. Then two of them were decomposed by annealing at $720 \mathrm{~K}$. One of decomposed 
samples was plastic deformed subsequently along 3 axes of [110] type. The mosaic-spread was $30^{\prime}-40^{\prime}$ for the quenched sample and $70^{\prime}-90^{\prime}$ for the aged samples.

The expected phase decomposition of the aged samples was confirmed by small angle neutron scattering. The resulting intensity distributions of scattered neutrons (the wavelength $=2.35 \AA$ ) are shown in Fig. 1 versus scattering angle. The intensity was not corrected for absorption; the illuminated sample volume was the same for all samples. The positions of intensity maxima were used for estimating the dimensions of different phase regions. They are of the order of magnitude of $80 \AA$ for the deformed sample and for not deformed sample they are more diffuse and smaller $(\approx 60 \AA)$.

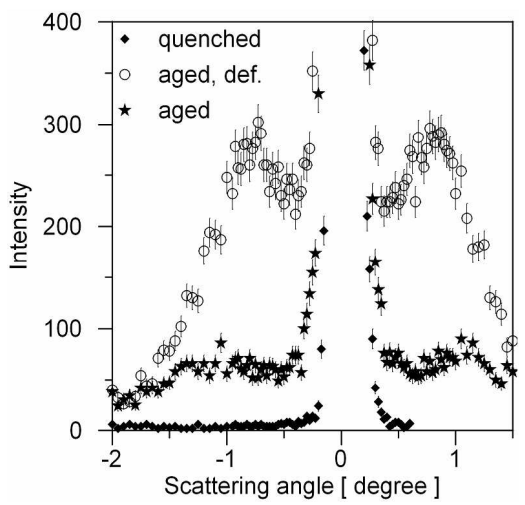

Fig. 1. The small angle neutron scattering results for the quenched, aged deformed, and aged not deformed sample.

At low temperatures all samples are antiferromagnetic of the F1 type, with magnetic moments oriented along one of the [100]-type axis. The Nèel temperature $T_{\mathrm{N}}$ is $\approx 300 \mathrm{~K}$ for the quenched sample and $\approx 465 \mathrm{~K}$ for the decomposed ones. The Nèel temperature of the quenched sample is much higher than known for homogeneous polycrystal samples with the same composition $T_{\mathrm{N}}=100 \mathrm{~K}[15]$, indicating that the sample is rather far from homogeneity. Probably the cooling rate during quenching $1 \mathrm{~cm}^{3}$ single-crystal was not sufficiently large and permitted partial decomposition. Crystal structure is fcc in the paramagnetic phase of all samples. All samples are polydomain in the antiferromagnetic phase.

In the magnetic phase tetragonal deformation takes place. The elastic neutron scattering at (200) RLP was measured to estimate deformation parameter. The examples of intensity distributions are presented in Fig. 2a for the quenched sample and in Fig. $2 b$ for the aged deformed one.

The measured distributions were analyzed assuming Gaussian shape of components. For the quenched sample the first maximum originating in domains with $c$ axis perpendicular to the scattering vector $\boldsymbol{Q}$ is placed at $|\boldsymbol{Q}|=4 \pi / a$ and the second maximum originating in domains with $c$ axis parallel to $\boldsymbol{Q}$ is placed at $|\boldsymbol{Q}|=4 \pi / c$. The structure analysis presented previously for the aged,

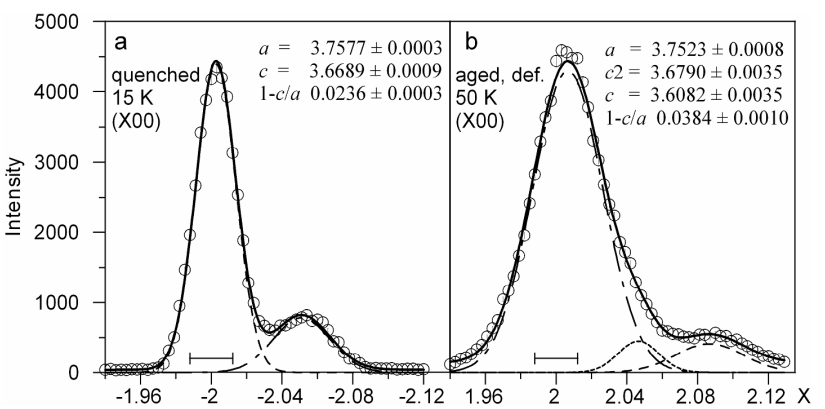

Fig. 2. Neutron intensity distributions measured at $(-2,0,0)$ RLP at $15 \mathrm{~K}$ on the quenched sample (a), and at $(2,0,0)$ RLP at $50 \mathrm{~K}$ on the aged deformed sample (b). Circles are the experimental results. The solid lines are the results of fitting and the dashed lines are the Gaussian components of the fitted curve. Horizontal bars represent the resolution FWHM.

deformed single-domain sample of the same alloy [7] was used to identify the origin of the scattering components for polydomain sample. The first maximum placed at $|\boldsymbol{Q}|=4 \pi / a$, originates in domains with $c$ axis perpendicular to $Q$ of the both sample regions with low and high $\mathrm{Cu}$ content. The second maximum at $|\boldsymbol{Q}|=4 \pi / c 2$ originates in domains with $c$ axis parallel to $\boldsymbol{Q}$ of the sample regions with high $\mathrm{Cu}$ content. The third maximum at $|\boldsymbol{Q}|=4 \pi / c$ originates in domains with $c$ axis parallel to $\boldsymbol{Q}$ of the sample regions with low $\mathrm{Cu}$ content. Below $50 \mathrm{~K}$ the resulting deformation parameter $1-c / a$ is $\approx 0.024$ for the quenched sample and $\approx 0.039$ for the phase of low $\mathrm{Cu}$ content of the aged deformed sample.

Elastic at (200) RLP and inelastic neutron scattering measurements were performed with three-axis spectrometer at MARIA reactor in Swierk. Spin waves were examined by neutron scattering in the vicinity of the (100) RLP. Measurements of intensity distributions of the scattered neutrons versus neutron energy transfer $I(E)$ and versus neutron momentum transfer $I(Q)$ were performed at several temperatures on both quenched and aged deformed samples and at $295 \mathrm{~K}$ on the aged not-deformed sample. The spectrometer energy resolution FWHM ranged from $1.1 \mathrm{meV}$, for energy transfer $0 \mathrm{meV}$ and energy of scattered neutrons $14.8 \mathrm{meV}$, to $3.6 \mathrm{meV}$, for energy transfer $20 \mathrm{meV}$ and energy of scattered neutrons $20 \mathrm{meV}$. Exemplary momentum resolution FWHM for energy transfer $12 \mathrm{meV}$ and energy of scattered neutrons $14.8 \mathrm{meV}$ was $0.073 \AA^{-1}$ for longitudinal scan and $0.13 \AA^{-1}$ for the transverse scan.

\section{Results}

Data analysis was performed assuming neutron scattering cross-section on $\mathrm{SW}$ in the following form:

$$
\begin{aligned}
& \frac{\mathrm{d}^{2} \sigma}{\mathrm{d} \Omega \mathrm{d} E} \propto \frac{E}{1-\exp (-E / k T)} \\
& \quad \times \frac{E(\boldsymbol{q}) \Gamma(\boldsymbol{q})}{\left[E^{2}-E(\boldsymbol{q})^{2}\right]^{2}+4 E^{2} \Gamma(\boldsymbol{q})^{2}},
\end{aligned}
$$

where energy broadening was assumed to depend linearly 
on spin wave vector

$$
\Gamma(\boldsymbol{q})=\Gamma_{0}+\Gamma_{1}|\boldsymbol{q}| .
$$

The convolution of the scattering cross-section with the spectrometer resolution function was fitted to the measured distributions. The background, normalization, and SW dispersion relation and energy broadening parameters were fitted. The energy gap value was determined from $I(E)$ distribution and used for analysis of $I(Q)$ distributions. The energy broadening was determined from a few $I(Q)$ distributions and obtained parameters were used for other distributions measured on the same sample at the same temperature.

Examples of $I(E)$ and $I(Q)$ distributions are presented in Figs. 3 and 4.

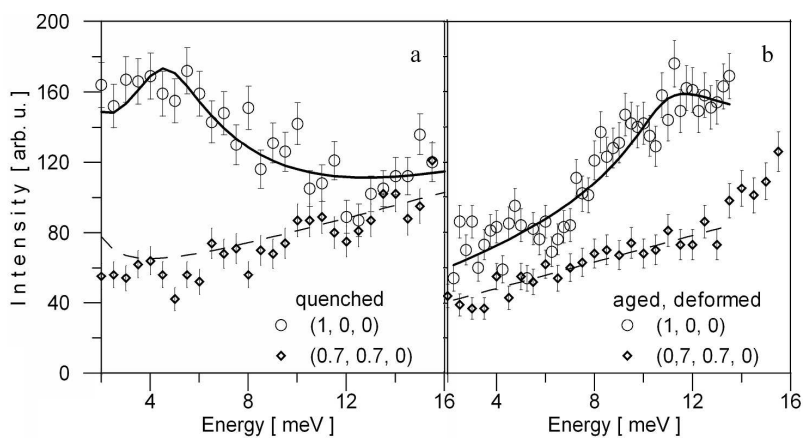

Fig. 3. Energy distributions of the scattered neutrons at the (100) RLP measured at $150 \mathrm{~K}$ on the quenched (a) and aged deformed (b) samples. Distributions at the $(0.7,0.7,0)$ indicate background.

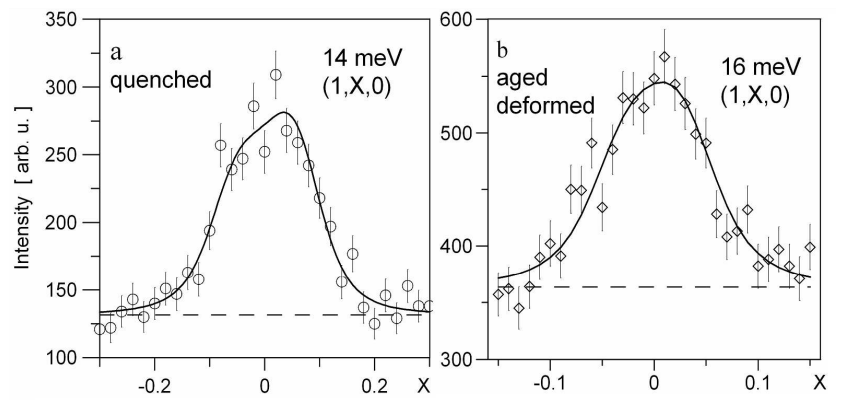

Fig. 4. Momentum distributions of the scattered neutrons at the (100) RLP measured at $150 \mathrm{~K}$ for energy transfer $14 \mathrm{meV}$ on the quenched sample (a) and for $16 \mathrm{meV}$ on the aged deformed sample (b).

Examples of the obtained dispersion relation are presented in Figs. 5, 6 and 7 for the quenched sample, the aged one after deformation and aged not deformed, respectively.

Determined parameters of the SW dispersion relation and energy broadening for all samples for different temperatures are collected in Table I.

At reduced temperature $T / T_{\mathrm{N}} \approx 0.65$ we obtained the values of the energy gap and velocity in the aged deformed sample twice bigger than in the quenched one.

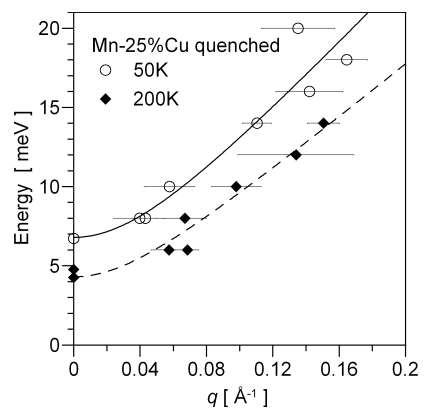

Fig. 5. Spin wave dispersion relation data, obtained at 50 and $200 \mathrm{~K}$ for the quenched sample. Lines represent dispersion relation: solid line $-T=50 \mathrm{~K}, E_{\mathrm{g}}=$ $6.8 \mathrm{meV}, v=112 \mathrm{meV} \AA$, dashed line $-T=200 \mathrm{~K}$, $E_{\mathrm{g}}=4.3 \mathrm{meV}, v=89 \mathrm{meV} \AA$.

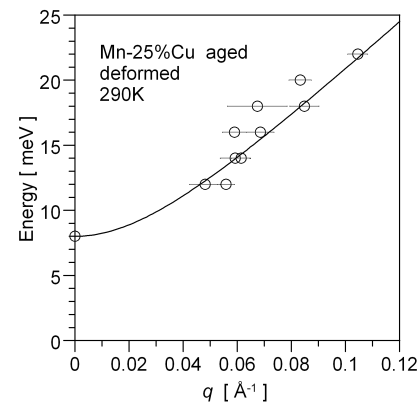

Fig. 6. Spin wave dispersion relation data obtained at $290 \mathrm{~K}$ for the aged, deformed sample. Line represents calculated dispersion relation for $E_{\mathrm{g}}=8 \mathrm{meV}$, $v=193 \mathrm{meV} \AA$.

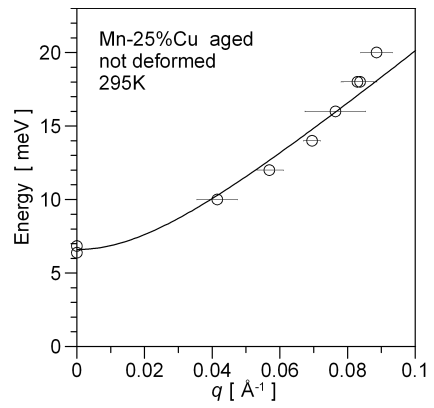

Fig. 7. Spin wave dispersion relation data obtained at $290 \mathrm{~K}$ for the aged sample. Line represents calculated dispersion relation for $E_{\mathrm{g}}=6.6 \mathrm{meV}, v=190 \mathrm{meV} \AA$.

TABLE I

Parameters of spin-wave dispersion-relation and energy broadening for $\mathrm{Mn}_{0.75} \mathrm{Cu}_{0.25}$ alloy after different thermal treatment.

\begin{tabular}{|c|c|c|c|c|c|c|}
\hline Sample & $T[\mathrm{~K}]$ & $\overline{T_{\mathrm{N}}[\mathrm{K}]}$ & $T / T_{\mathrm{N}}$ & $E_{\mathrm{g}}[\mathrm{meV}]$ & $\overline{v[\mathrm{meV} \AA]}$ & $\Gamma_{1}[\mathrm{meV} \AA]$ \\
\hline quenched & 50 & $\approx 300$ & 0.17 & $6.0 \pm 0.6$ & $121 \pm 13$ & $99 \pm 33$ \\
\hline quenched & 100 & $\approx 300$ & 0.33 & $5.8 \pm 0.4$ & $133 \pm 12$ & $109 \pm 37$ \\
\hline quenched & 150 & $\approx 300$ & 0.50 & $4.5 \pm 0.4$ & $120 \pm 14$ & $62 \pm 15$ \\
\hline quenched & 200 & $\approx 300$ & 0.67 & $3.7 \pm 0.3$ & $89 \pm 8$ & $82 \pm 25$ \\
\hline aged & 295 & 450 & 0.66 & $6.6 \pm 0.2$ & $190 \pm 12$ & $72 \pm 18$ \\
\hline $\begin{array}{l}\text { aged and } \\
\text { deformed }\end{array}$ & 150 & 465 & 0.32 & $10.9 \pm 0.4$ & $214 \pm 28$ & \\
\hline $\begin{array}{l}\text { aged and } \\
\text { deformed }\end{array}$ & 295 & 465 & 0.63 & 8.0 & $193 \pm 11$ & \pm 18 \\
\hline
\end{tabular}


For the aged not deformed sample the value of the energy gap is smaller than in the deformed sample.

\section{Discussion and conclusions}

The comparison of the previously published data on spin-wave dispersion-relation parameters in $\mathrm{Mn}-\mathrm{Cu}$ alloys is given in Table II. The quoted results were obtained for the quenched samples excluding the last aged one.

The scatter of the SW velocity values (Table II) for different compositions and temperatures is rather small compared to that of the SW energy gap value. This fact is consistent with the prediction that SW velocity is determined mainly by the electron Fermi velocity and energy gap depends mainly on the sub-lattice magnetization.

TABLE II

Parameters of spin-wave dispersion-relation and energy broadening for $\mathrm{Mn}_{1-x} \mathrm{Cu}_{x}$ alloys with different $\mathrm{Cu}$ content $x$, previous data. For $x=0.25$ aged for $1 \mathrm{~h}$.

\begin{tabular}{c|c|c|c|c|c|c|c}
\hline \hline $\mathrm{x}$ & $T[\mathrm{~K}]$ & $T_{\mathrm{N}}[\mathrm{K}]$ & $T / T_{\mathrm{N}}$ & $E_{\mathrm{g}}[\mathrm{meV}]$ & $v[\mathrm{meV}]$ & $\Gamma_{1}[\mathrm{meV}]$ & Ref. \\
\hline 0.1 & 113 & 465 & 0.243 & $12.0 \pm 0.8$ & $210 \pm 8$ & $118 \pm 30$ & {$[8]$} \\
0.1 & 295 & 465 & 0.63 & $8.7 \pm 0.4$ & $182 \pm 3$ & $117 \pm 14$ & {$[8]$} \\
0.1 & 295 & 465 & 0.63 & 7.5 & $256 \pm 1$ & $397 \pm 5$ & {$[9]$} \\
0.15 & & & & 5 & 210 & 70 & {$[10]$} \\
0.18 & 10 & 400 & 0.025 & $7.8 \pm 0.2$ & $224 \pm 3$ & $101 \pm 3$ & {$[12]$} \\
0.18 & 100 & 400 & 0.25 & & $80 \pm 20$ & & {$[11]$} \\
0.18 & 295 & 355 & 0.83 & $5.5 \pm 1$ & $170 \pm 26$ & & {$[16]$} \\
0.25 & 295 & 410 & 0.72 & $4 \pm 1$ & $190 \pm 20$ & & {$[17]$}
\end{tabular}

The obtained values of the parameters of the SW dispersion relation for the decomposed sample are very close to those found for the quenched $\mathrm{Mn}_{0.9} \mathrm{Cu}_{0.1}$ alloy by Wiltshire et al. [8]. The value of SW velocity found by Fernandez-Baca et al. for the same composition [9] is larger, but their result seems to be connected with much higher energy broadening. Thus our result should be compared to that of [8] rather than to [9]. The similar value of the energy gap obtained in both [8] and [9] cases and for the aged samples confirms that $\mathrm{Cu}$ concentration is close to 0.1 in the sample regions with the $\mathrm{F} 1$ order. It seems to be well established that after spinodal decomposition spin waves are observed only in the regions of low $\mathrm{Cu}$ concentration and they are not influenced by the neighborhood of high $\mathrm{Cu}$ concentration regions, at least for long wavelengths.

In detail the influence of decomposition on SW parameters is not so clear. For the aged not deformed sample the value of the energy gap is smaller than in the deformed sample, which is consistent with smaller lattice deformation in this sample and is probably caused by smaller degree of decomposition that can be deduced from lower intensity of the small angle neutron scattering (Fig. 1). In our old unpublished data for $1 \mathrm{~h}$ aged sample [17] the gap is nearly as small as in the quenched sample. The SW velocity does not differ for all aged samples, but for the quenched sample it is significantly lower.

Most interesting result of the present investigation is that the SW velocity for the quenched sample is lower than observed in any other manganese alloy. The reason of the lowering of velocity is probably not SW scattering on impurities, since energy broadening is not larger than for most other investigated alloys. In terms of band model the result may suggest significant changes in the electron band structure (the Fermi velocity) of $\mathrm{Mn}_{0.75} \mathrm{Cu}_{0.25}$ alloy compared to other manganese alloys.

\section{References}

[1] J.H. Smith, E.R. Vance, J. Appl. Phys. 40, 4853 (1969).

[2] E.Z. Vintaikin, V.B. Dmitriev, V.A. Udovenko, Fiz. Met. Metallov. 46, 790 (1978).

[3] B.D. Gaulin, S. Spooner, Y. Morii, Phys. Rev. Lett 59, 668 (1987).

[4] J. Jankowska, K. Mikke, E.Z. Vintaikin, V.A. Udovenko, J. Magn. Magn. Mater. 27, 41 (1982).

[5] Y. Shiozaki, Y. Nakai, J. Phys. Soc. Jpn. 47, 817 (1979).

[6] E.Z. Vintaikin, V.B. Dmitriev, V.A. Udovenko, Fiz. Met. Metallov. 59, 742 (1985).

[7] J. Jankowska-Kisielińska, K. Świderska, K. Mikke, Acta Phys. Pol. 117, 582 (2010).

[8] M.C.K. Wiltshire, M.M. Elcombe, C.J. Howard, J. Phys. F 15, 1595 (1985).

[9] J.A. Fernandez-Baca, M.E. Hagen, R.M. Nicklow, Y. Tsunoda, S.M. Hayden, J. Magn. Magn. Mater. 104-107, 699 (1992).

[10] Y. Tsunoda, Y. Nakai, Solid State Commun. 34, 413 (1980).

[11] K. Mikke, J. Jankowska, E. Jaworska, J. Magn Magn. Mater. 31-34, 125 (1983).

[12] K. Mikke, T.M. Holden, J.A. Fernandez-Baca, E. Fawcett, J. Jankowska-Kisielińska, in: Proc. Int. Conf. "Physics of Transition Metals", Darmstadt, 1992, Eds. P.M. Oppeneer, J. Kübler, World Sci., Singapore 1993, p. 659.

[13] M.J. Gillan, J. Phys. F: Met. Phys. 3, 1874 (1973).

[14] R.S. Fishman, S.H. Liu, Phys. Rev. 59, 8681 (1999).

[15] E.Z. Vintaikin, V.B. Dmitriev, V.A. Udovenko, Fiz. Met. Metallov. 44, 1023 (1977).

[16] K. Mikke, J. Jankowska-Kisielińska, B. Hennion, Physica B 350, e75 (2004).

[17] K. Mikke, J. Jankowska-Kisielińska, E.Z. Vintaikin, V.A. Udovenko, Rap. IBJ 12/XIV/P/79, 1979. 